\title{
Frequency of hand-to-head, -mouth, -eyes, and -nose contacts for adults and children during eating and non-eating macro-activities
}

\author{
Amanda M. Wilson $\mathbb{D}^{1} \cdot$ Marc P. Verhougstraete $^{1} \cdot$ Paloma I. Beamer $^{1} \cdot$ Marco-Felipe King $^{2} \cdot$ Kelly A. Reynolds $^{1} \cdot$ \\ Charles P. Gerba ${ }^{1,3}$
}

Received: 11 March 2020 / Revised: 3 June 2020 / Accepted: 1 July 2020 / Published online: 15 July 2020

(c) The Author(s) 2020. This article is published with open access

\begin{abstract}
Hand-to-face contacts are important for estimating chemical and microbial exposures. Few studies describe children's handto-eye or -nose contacts or adults' hand-to-face contacts. The study objective was to characterize hand-to-head (mouth, eyes, nose, and other) contacts for children in a daycare and adults in multiple locations. Macro-activities and sequences of handto-face contacts were recorded for 263 people observed for $30 \mathrm{~min}$ each. Statistically significant differences between locations, males and females, adults and children, and during eating and non-eating macro-activities were evaluated. Discrete Markov chains were fit to observed contact sequences and compared among adults and children during eating and non-eating macro-activities. No significant differences in contact frequency were observed between males and females with the exception of hand-to-nose contacts. Children tended to touch the mouth, eyes, and nose more frequently than adults during non-eating macro-activities. Significant differences in contact frequency were observed between locations. Transitional probabilities indicated that children make repetitive mouth, eye, and nose contacts while adults frequently transition to contacts of the head other than the mouth, eyes, or nose. More data are needed to evaluate the effect of age on adults' contact frequencies and to confirm lack of statistically significant differences between adults and children during eating macroactivities.
\end{abstract}

\section{Introduction}

Hand-to-face contacts are important behaviors that can result in dermal and non-dietary exposures. Hand-to-mouth contacts are especially of concern when considering exposures to chemicals (e.g., lead [1] and pesticides [2]) and microbial pathogens with fecal-oral transmission. Hand-to-eye and

Supplementary information The online version of this article (https:// doi.org/10.1038/s41370-020-0249-8) contains supplementary material, which is available to authorized users.

$\square$ Amanda M. Wilson

apfeifer@email.arizona.edu

1 Department of Community, Environment \& Policy, Mel and Enid Zuckerman College of Public Health, University of Arizona, Tucson, AZ, USA

2 School of Civil Engineering, University of Leeds, Woodhouse Lane, Leeds LS29JT, UK

3 Department of Environmental Science, College of Agriculture and Life Sciences, University of Arizona, Tucson, AZ, USA -nose contacts are of concern especially for respiratory pathogens, such as rhinovirus, influenza viruses, and coronaviruses [3-6]. Frequencies of contacts to the face are often used in exposure modeling to quantify a dose and subsequent health risk [7-11]. However, hand-to-face contact data are limited, especially for adults. In several quantitative microbial risk assessments, the frequency of hand-to-mouth, -eyes, and/or -nose contacts were informed by a study of ten student volunteers [12]. With a lack of micro-activities (second-by-second behaviors) for adults, several models also utilize contact frequencies of $7-12$ year olds $(n=18)$, based on the hypothesis that their mouthing behaviors and other hand-to-object contacts may approach those expected of adults [7, 13].

While micro-activity data specific for adults are sparse, contact frequencies, especially with the face, appear as the most influential parameters in several sensitivity analyses of exposure models [11, 14, 15]. In a rotavirus fomitemediated exposure model for children, frequency of fomite-to-mouth contacts was the second most influential model parameter [11]. In a risk assessment of viral pathogens spread in office buildings, frequency of hand-to-mouth 
contacts was the second most influential parameter for rotavirus infection risk estimation [14]. For a respiratory virus infection risk, the second most influential parameter was frequency of hand-to-nose contacts [14]. Hand-tomouth contact frequency has also been an influential parameter for dust ingestion estimates, [15] and hand-tomouth contacts are included in US EPA's Exposure Factors Handbook in a larger discussion of the importance of micro-activity patterns [16]. Only one identified study provided mouthing behaviors (hand-to-mouth or objectto-mouth) for adults $[12,16]$. Another study quantified frequency of face contacts, with mucosal and nonmucosal membrane areas, for medical students $(n=26)$ [17]. However, it is uncertain how these contact frequencies compare to adults in other locations. Increasing available data and information regarding differences in hand-to-face frequency for males vs. females and by age is an important step in reducing uncertainties in current exposure science and risk assessment.

The objective of this study was to compare frequency and sequence of hand-to-head, -mouth, -eyes, -nose contacts between males and females, adults and children, and during dietary and non-dietary macro-activities. This study expands upon current available hand-to-face contact data for adults, which is limited to office and university lecture settings $[12,17]$. In this study, micro-activities for adults were collected in multiple locations: airport, bar, church, classroom, food court, museum, public library, university library, and a sporting event.

\section{Methods}

\section{Observations}

Per observation, a person was observed for a 30-min period in daycare, airport, bar, church, classroom, food court, museum, public library, university library, and sporting event environments. Subjects were not explicitly aware of being observed and were chosen at random in the location by the observer. If it was clear that the observer was noticed or if the person being observed left the location before the 30-min observation period had ended, this entire observation was excluded from the study. The observer made a best estimate of the gender and age of the participant (male/female), and there was no interaction between the observer and the participants.

In total, 263 people were observed: 99 adult males, 100 adult females, 32 male children, and 32 female children. Each observation was categorized as eating (i.e., person was eating food) or non-eating (i.e., person was not eating food, regardless of drinking activities). Contacts with the nose, mouth, or eyes, along with other areas, including cheek, forehead, temples, hair, ears, and neck, were recorded by hand where each new contact was listed in chronological order on the activity observation form.

Only areas of the nose, mouth, and eyes thought to potentially lead to infection were counted towards nose, mouth, and eye contact frequencies. Specifically, nose contacts were defined as contact with the inner or outer nose (nostril area, excluding nasal bone or bridge of the nose) surfaces and under the nose. Mouth contacts were defined as contact with the lips, teeth, or inner mouth surfaces. Contact with the eyes included contact with the corner of the eye, the eyelid, conjunctiva or an eye rub. Contacts with the head included any of these contacts or contacts with the cheek, forehead, temples, hair, chin, ears, or neck. Contacts defined as "other" excluded the mouth, eyes, or nose and included contacts with the cheek, temples, hair, chin, ears, or neck. Although some contacts, such as contact to the outside of the nose, may not directly result in a dose or exposure for all cases, due to challenges in the angle of the observer, we assumed any contact with these surfaces pertaining to the nose, mouth, or eye surfaces could result in a dose. Each recorded touch began when contact was made and finished at the first lift of the hand from the contacted facial surface.

The time (hour and minute) for each contact was recorded. Contacts with parts of the head other than the mouth, eyes, or nose were grouped together in the analysis as "other." The University of Arizona Office for Human Research Protections determined human subjects review was not required (Protocol Number: 1911145109). One observer conducted all the observations in 2001 and has since passed away. Observation forms were translated to a digital spreadsheet format by one researcher. This researcher and another researcher separately chose entries from the digital spreadsheet at random and checked agreement with the original observation forms. Descriptive statistics of contact frequencies and transitional probabilities for contact sequences are reported here to inform exposure estimation and risk assessment.

\section{Analysis of contact frequencies}

Statistical analysis was conducted with R statistical software (version 3.6.0, R Core Team, 2019). We tested for statistically significant $(\alpha=0.05)$ differences in frequency of all hand-to-head, -mouth, -eyes, -nose, and -other contacts using Wilcoxon rank sum tests for males vs. females, adults vs. children, and during eating and non-eating macroactivities. Differences between children and adults were investigated to address the current knowledge gap that children's behaviors are not equivalent to those of adults [18]. Because the same observation methods for adults 
and children were used in this study, this allowed for comparisons less influenced by differences in observational methods. Descriptive statistics reported in tables were stratified based on statistically significant differences. To test for statistically significant differences in frequencies across locations, Kruskal-Wallis tests $(\alpha=0.05)$ followed by Dunn's post hoc tests with a Hochberg adjustment for multiple comparisons $(\alpha=0.025)$ were used. To remove potential confounding of eating behavior differences on contact differences across location, differences in frequencies across location were tested for eating macroactivity and non-eating macro-activity observations separately. The range, arithmetic mean, standard deviation, and 5th, 25th, 50th, 75th, 95th, and 99th quantiles of contact frequencies were calculated.

\section{Analysis of contact sequence}

Significant differences found in contact frequency were used to guide stratification of contact sequences. The function markovchainFit from the $\mathrm{R}$ package, "markovchain," was used with a maximum likelihood estimation method to estimate transitional probabilities between contacts with the mouth, eyes, nose, or other (any contact with the face or head other than the mouth, eyes, or nose) [19]. Transitions in this case do not account for contact duration and represent transitions between hand-to-face contacts only. For example, a contact with the nose followed by a contact to a nearby object and another contact to the nose would represent a nose to nose contact transition. Estimates were used to inform heat maps for visual comparisons, and
95\% confidence intervals of estimates are available in supplemental material (Table S1).

\section{Results}

\section{Contact frequencies}

\section{Comparison of males and females}

There were no statistically significant differences between contact frequencies of males $(n=131)$ and females $(n=$ 132) other than for hand-to-nose contacts, which was significant but with a $p$ value of $p=0.049$ (Fig. 1). When stratified by age, significant differences in hand-to-nose contact frequency between male and female participants were seen for adults ( $p=0.0014)$, but not for children $(p=$ 0.36) (Supplementary Fig. S1). Males tended to touch their nose more frequently than females, with a median contact frequency of 4.0 contacts/h (standard deviation $(\mathrm{SD})=$ $2.83)$ as opposed to 2.0 contacts/h $(\mathrm{SD}=2.77)$. Among adults, specifically, males and females touched their nose with a median contact frequency of 2.00 contacts/h. However, the average contact frequency for adult males was 2.97 contacts/h, while it was 2.06 contacts/h for females.

\section{Comparison of adults and children}

All contact frequencies were significantly different $(p<0.05)$ between adults $(n=199)$ and children $(n=64)$ (Fig. 2). Children tended to touch their head, mouth, eyes, and nose
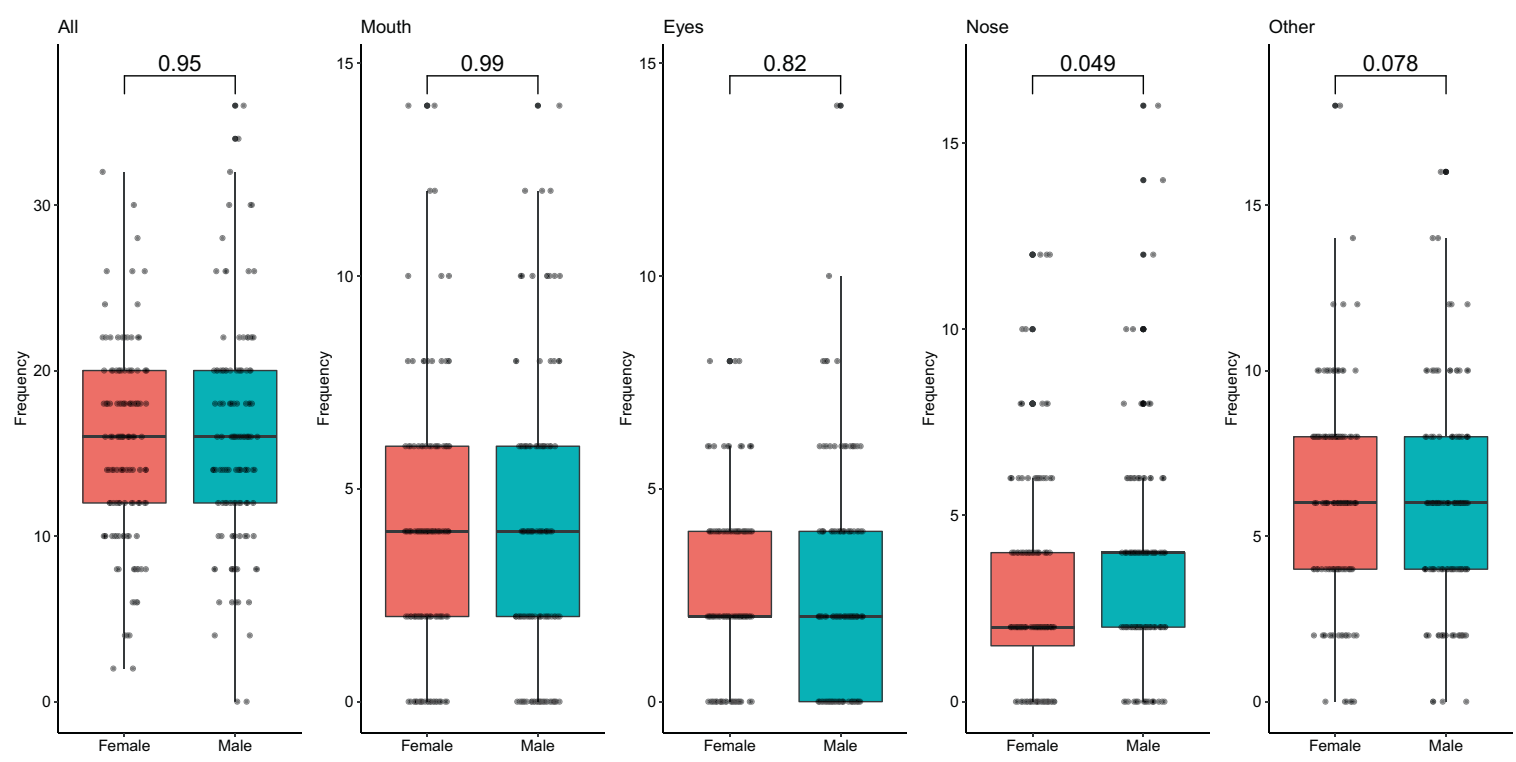

Fig. 1 Comparison of contact frequencies among locations during non-eating macro-activities. Post-hoc analysis results for a all face contacts, $\mathbf{b}$ eye contacts, and $\mathbf{c}$ other contacts (not -mouth, -eyes, or -nose). Dunn's test $p$ values with Hochberg adjustment are indicated by color, where dark purple relates to non-statistically significant differences. 

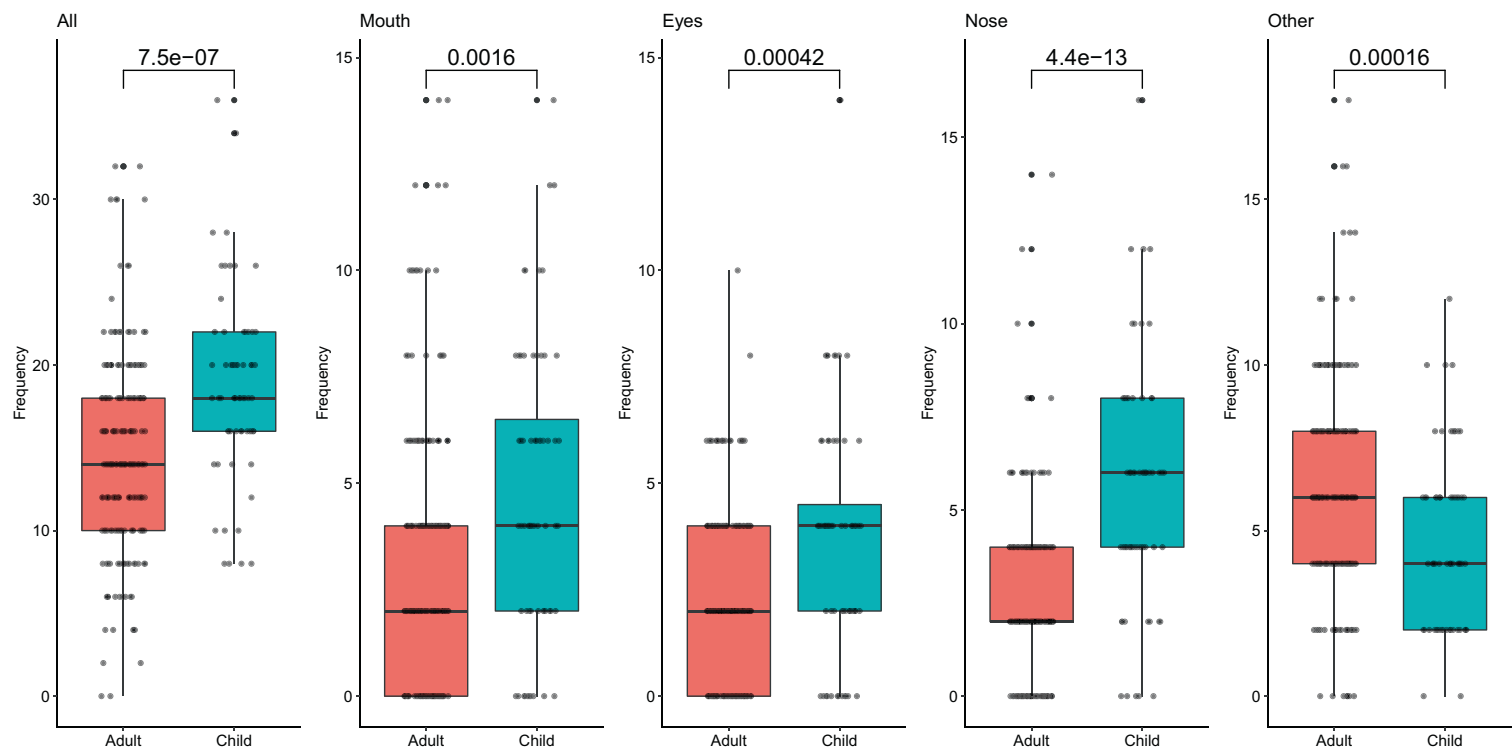

Fig. 2 Contact frequency (contacts/h) of hand-to-head (all), -mouth, -eyes, -nose, and -other contacts for adults and children. Statistical comparisons were calculated using a Wilcoxon rank sum test and can be seen over bars.

more frequently than adults, while adults made contacts with other parts of the head more frequently than children (Fig. 2). Children touched their head with a median frequency of 18.1 contacts/h $(\mathrm{SD}=5.6)$ while adults touched their head with an average frequency of 14.0 contacts/h $(\mathrm{SD}=5.9)$ (Table 1). Children touched their mouths with a median frequency of 4.0 contacts/h $(\mathrm{SD}=3.4)$, while adults touched their mouths with a median frequency of 2.0 contacts/h $(\mathrm{SD}=3.1)$ (Table 1$)$. Children touched their eyes with a median frequency of 4.0 contacts/h $(\mathrm{SD}=2.8)$, and adults touched their eyes with a median frequency of 2.0 contacts/h $(\mathrm{SD}=2.0)$ (Table 1$)$. Children touched their nose with a median frequency of 6.0 contacts/h $(\mathrm{SD}=3.2)$, while adults touched their nose with a median frequency of 2.0 contacts/h $(\mathrm{SD}=2.2)$ (Table 1$)$. Children touched parts of the head other than the mouth, eyes, or nose with a median frequency of 4.0 contacts/ $h(S D=2.6)$, and adults touched other parts of the head with a median frequency of 6.0 contacts/h $(\mathrm{SD}=3.2)$ (Table 1).

\section{Comparison of eating vs. non-eating macro-activity contexts}

Frequency of hand-to-head (all) and -mouth contacts were significantly different $(p<0.05)$ during eating macro-activities $(n=22)$ vs. non-eating $(n=241)$ macro-activities, with greater hand-to-head and -mouth contacts during eating (Fig. 3). During eating, the median frequency of hand-tomouth contacts was 7 contacts/h $(\mathrm{SD}=3.9)$, while during non-eating macro-activities, the median frequency of hand-tomouth contacts was 4 contacts/h $(\mathrm{SD}=2.9)$. Contact frequency summary statistics were stratified by age and by eating vs non-eating macro-activity behavior (Table 1).
When comparisons between children and adults were stratified by eating or non-eating activities, all contact frequencies were significantly different $(p<0.05)$ between adults and children for non-eating activities. However, during eating activities, significant differences were not seen (Supplementary Fig. S2). Few children were observed during eating macro-activities $(n=3)$, however.

During eating behaviors, the median frequency of handto-mouth contacts for children $(8$ contacts/h) was greater than that of adults ( 6 contacts/h) (Table 1$)$, but there was no statistically significant difference (Supplementary Fig. S2). The 75th percentile of hand-to-mouth contact frequency for adults $(11$ contacts/h) was greater than that of children $(9$ contacts $/ \mathrm{h}$ ), and a wider range of contact frequency was observed for adults (Table 1). A similar pattern was seen in differences between adults and children in hand-to-eye and -nose contacts. A wider range in contact frequencies were seen for adults, where adults at higher percentiles touched their eyes and nose more than children at the same percentiles during eating activities (Table 1). During noneating activities, children tended to touch the head, mouth, eyes, and nose more frequently than adults, while adults tended to touch parts of the head other than the mouth, eyes, or nose with greater frequency (Table 1).

\section{Comparison of locations}

No significant differences were observed in contact frequencies among locations during eating macro-activities. For non-eating macro-activities, statistically significant differences among locations were observed for hand-to-head (all) $\left(\chi^{2}=49.656, \mathrm{df}=9, p\right.$ value $\left.=1.3 \times 10^{-7}\right)$, hand-to-mouth 
Table 1 Summary statistics of contact frequency (contacts/h) of hand-to-head (all), -mouth, -eyes, -nose, and -other for adults vs. children during eating and non-eating macro-activities including 5th, 25th, 50th, 75th, 95th, and 99th percentiles.
Age

Facial feature Range (Min, Max) Mean \pm SD 5th 25th 50th 75th 95th 99th

Eating macro-activity

Children $(n=3)$

Mouth

$(4.0,10.0)$

Eyes

$(2.0,4.0)$

Nose

$(0.0,6.0)$

Other

$(2.0,2.0)$

All

$(8.0,20.0)$

Adults $(n=19)$

$(2.0,14.0)$

Eyes

$(0.0,10.0)$

Nose

$(0.0,8.0)$

Other

$(0.0,16.0)$

All

$(6.0,32.0)$

Non-eating macro-activity

Children $(n=61) \quad$ M

Eyes

$(0.0,14.0)$

Nose

$(0.0,14.0)$

Other

$(0.0,16.0)$

All

$(0.0,12.0)$

$(8.0,36.0)$

Adults $(n=180) \quad$ Mouth

$(0.0,10.0)$

Eyes

$(0.0,8.0)$

Nose

$(0.0,14.0)$

Other

$(0.0,18.0)$

All

$(0.0,30.0)$

All macro-activities

$\begin{array}{lll}\text { Children }(n=64) & \text { Mouth } & (0.0,14.0) \\ & \text { Eyes } & (0.0,14.0) \\ & \text { Nose } & (0.0,16.0) \\ & \text { Other } & (0.0,12.0) \\ \text { Adults }(n=199) & \text { All } & (8.0,36.0) \\ & \text { Mouth } & (0.0,14.0) \\ & \text { Eyes } & (0.0,10.0) \\ & \text { Nose } & (0.0,14.0) \\ & \text { Other } & (0.0,18.0) \\ & \text { All } & (0.0,32.0)\end{array}$

$\begin{array}{rrrrrrr}7.3 \pm 3.1 & 4.4 & 6.0 & 8.0 & 9.0 & 9.8 & 10.0 \\ 2.7 \pm 1.2 & 2.0 & 2.0 & 2.0 & 3.0 & 3.8 & 4.0 \\ 2.0 \pm 3.5 & 0.0 & 0.0 & 0.0 & 3.0 & 5.4 & 5.9 \\ 2.0 \pm 0.0 & 2.0 & 2.0 & 2.0 & 2.0 & 2.0 & 2.0 \\ 14.0 \pm 6.0 & 8.6 & 11.0 & 14.0 & 17.0 & 19.4 & 20.0 \\ 7.7 \pm 4.1 & 2.0 & 4.0 & 6.0 & 11.0 & 14.0 & 14.0 \\ 2.1 \pm 2.8 & 0.0 & 0.0 & 0.0 & 4.0 & 6.4 & 9.3 \\ 2.4 \pm 2.2 & 0.0 & 1.0 & 2.0 & 4.0 & 6.2 & 7.6 \\ 7.6 \pm 4.5 & 1.8 & 4.0 & 8.0 & 10.0 & 14.2 & 15.6 \\ 19.8 \pm 7.8 & 7.8 & 14.0 & 20.0 & 24.0 & 32.0 & 32.0\end{array}$

$\begin{array}{lllllll}4.7 \pm 3.4 & 0.0 & 2.0 & 4.0 & 6.0 & 10.0 & 12.8\end{array}$

$\begin{array}{lllllll}3.7 \pm 2.8 & 0.0 & 2.0 & 4.0 & 6.0 & 8.0 & 10.4\end{array}$

$\begin{array}{lllllll}5.7 \pm 3.1 & 2.0 & 4.0 & 6.0 & 8.0 & 12.0 & 13.6\end{array}$

$\begin{array}{lllllll}4.8 \pm 2.6 & 2.0 & 2.0 & 4.0 & 6.0 & 10.0 & 10.8\end{array}$

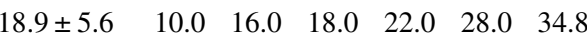

$2.9 \pm 2.5 \quad 0.0 \quad 0.0 \quad 2.0 \quad 4.0 \quad 8.0 \quad 10.0$

$2.4 \pm 1.9 \quad 0.0 \quad 0.0 \quad 2.0 \quad 4.0 \quad 6.0 \quad 6.0$

$\begin{array}{lllllll}2.5 \pm 2.2 & 0.0 & 2.0 & 2.0 & 4.0 & 6.0 & 10.4\end{array}$

$\begin{array}{lllllll}6.2 \pm 3.0 & 2.0 & 4.0 & 6.0 & 8.0 & 10.0 & 14.4\end{array}$

$\begin{array}{lllllll}14.0 \pm 5.4 & 5.9 & 10.0 & 14.0 & 18.0 & 22.0 & 26.8\end{array}$

$\begin{array}{rrrrrrr}4.8 \pm 3.4 & 0.0 & 2.0 & 4.0 & 6.5 & 10.0 & 12.7 \\ 3.7 \pm 2.8 & 0.0 & 2.0 & 4.0 & 4.5 & 8.0 & 10.2 \\ 5.5 \pm 3.2 & 0.0 & 4.0 & 6.0 & 8.0 & 11.7 & 13.5 \\ 4.7 \pm 2.6 & 2.0 & 2.0 & 4.0 & 6.0 & 9.7 & 10.7 \\ 18.7 \pm 5.6 & 8.3 & 16.0 & 18.1 & 22.0 & 27.7 & 34.7 \\ 3.4 \pm 3.1 & 0.0 & 0.0 & 2.0 & 4.0 & 10.0 & 12.0 \\ 2.3 \pm 2.0 & 0.0 & 0.0 & 2.0 & 4.0 & 6.0 & 6.0 \\ 2.5 \pm 2.2 & 0.0 & 2.0 & 2.0 & 4.0 & 6.0 & 10.0 \\ 6.3 \pm 3.2 & 2.0 & 4.0 & 6.0 & 8.0 & 12.0 & 16.0 \\ 14.6 \pm 5.9 & 6.0 & 10.0 & 14.0 & 18.0 & 22.2 & 30.0\end{array}$

$\left(\chi^{2}=26, \mathrm{df}=9, \quad p\right.$ value $\left.=0.0020\right)$, hand-to-eyes $\left(\chi^{2}=\right.$ 17.016, $\mathrm{df}=9, p$ value $=0.048)$, and hand-to-other $\left(\chi^{2}=\right.$ $39.459, \mathrm{df}=9, p$ value $\left.=9.5 \times 10^{-6}\right)$ contacts. There were no statistically significant differences seen between locations in hand-to-nose contact frequency. Multiple statistically significant differences between frequencies by location were seen in post hoc tests (Fig. 4). For hand-to-head contacts ("all" contacts), significant differences were observed between the airport and classroom, airport and zoo, church and museum, classroom and museum, classroom and university, museum and sporting event, and museum and zoo (Fig. 4a). For hand-to-mouth contacts, no significant differences were observed. For hand-to-eye contacts, a significant difference between the university library and classroom was observed (Fig. 4b). For hand-to-other contacts, significant differences were seen between the classroom and airport, church and museum, classroom and museum, classroom and public library, and museum and sporting event (Fig. 4c).

\section{Contact sequences}

Adults and children had notably different transitional probabilities for hand-to-head contacts, where adults tended to transition to hand-to-other contacts with greater probability from any type of hand-to-head contact, while children tended to make repetitive contacts with the mouth, eyes, or nose (Fig. 5a, b). Differences were also observed between eating and non-eating activities for adults, where during eating activities repetitive hand-to-mouth contacts were likely in addition to transitions from any hand-to-head 

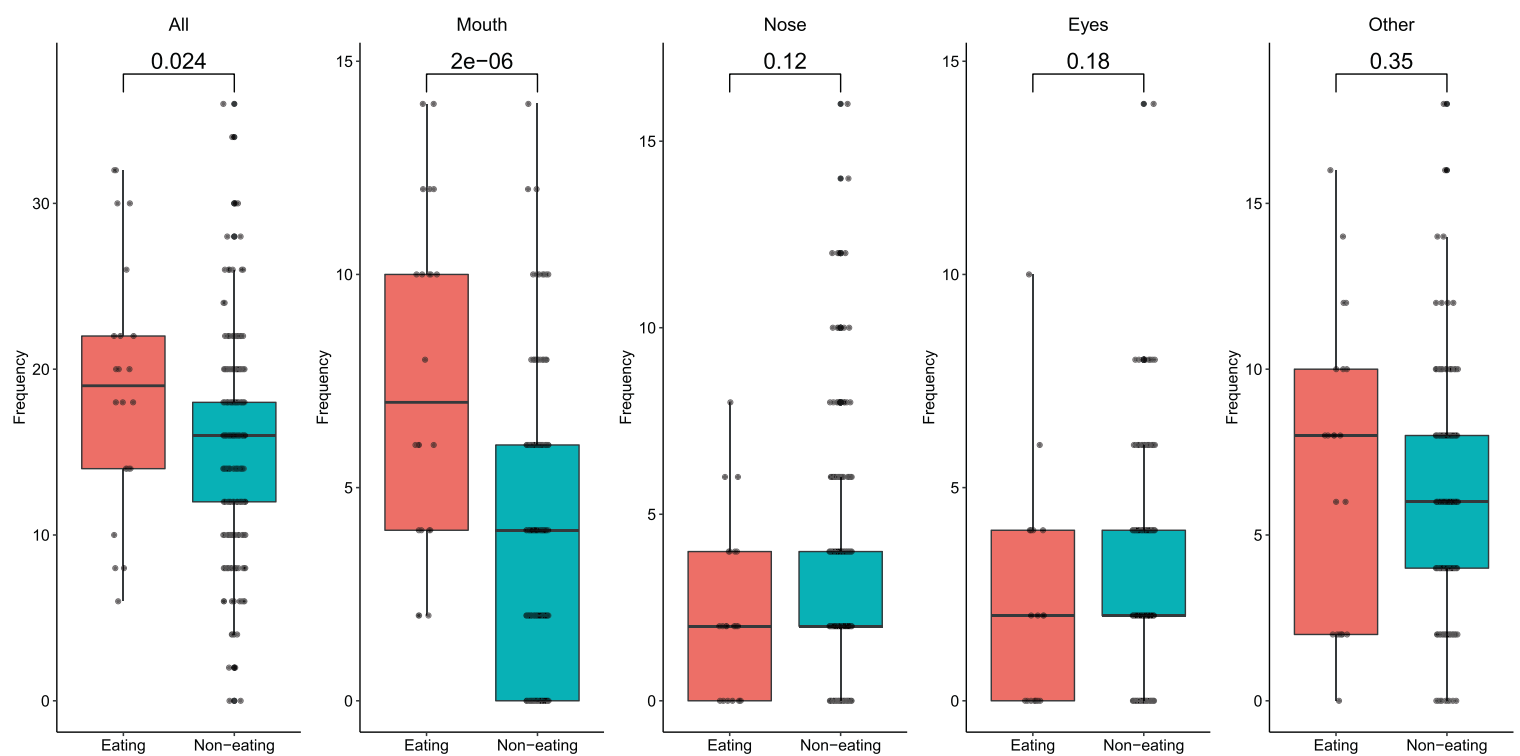

Fig. 3 Comparison of frequencies (contacts/h) of hand-to-head (all), -mouth, -eyes, -nose, and -other contacts during eating and non-eating macro-activity behavior. Statistical comparisons were calculated using a Wilcoxon rank sum test and can be seen over bars.

Fig. 4 Post-hoc analysis results of location comparison. Dunn's test $p$ values with Hochberg adjustment for comparison of contact frequencies among locations during non-eating macro-activities are shown for a all face contacts, $\mathbf{b}$ eye contacts, and $\mathbf{c}$ other contacts (not -mouth, -eyes, or -nose. Dark purple relates to nonstatistically significant differences.

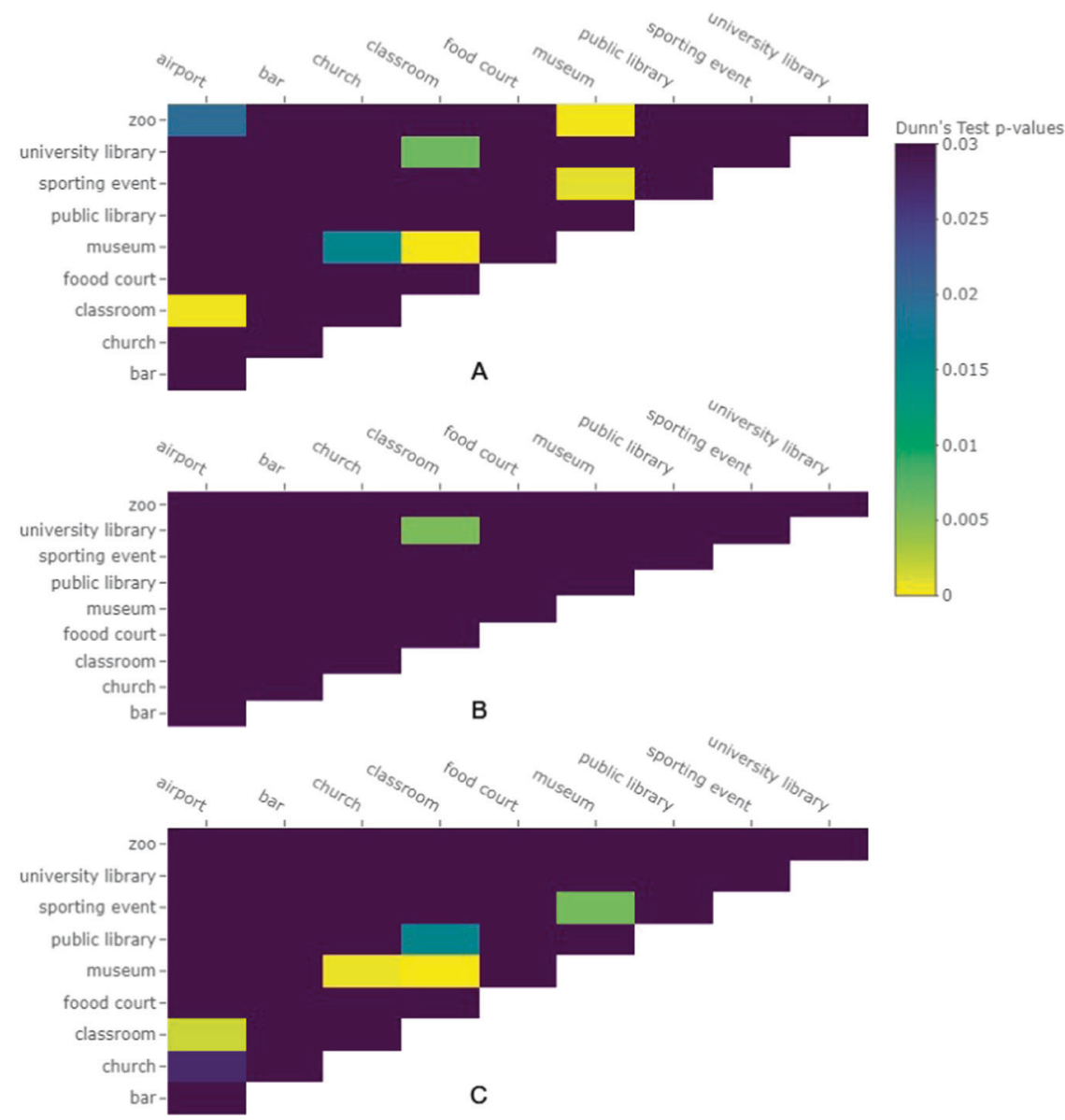

contact to a hand-to-mouth contact or a hand-to-other contact (Fig. 5c, d). During non-eating activities, the transitional probabilities were similar to those for all adults grouped together, where any hand-to-head contact was likely to transition to a hand-to-other contact (contacts with parts of the head or face other than the mouth, eyes, or nose) 
Fig. 5 Heat maps of transitional probabilities. Transitional probabilities (the probability of transitioning from one contact type to another) are shown for a adults and b children, $\mathbf{c}$ adults during eating, $\mathbf{d}$ adults during noneating, e children during eating, f children during non-eating.
A
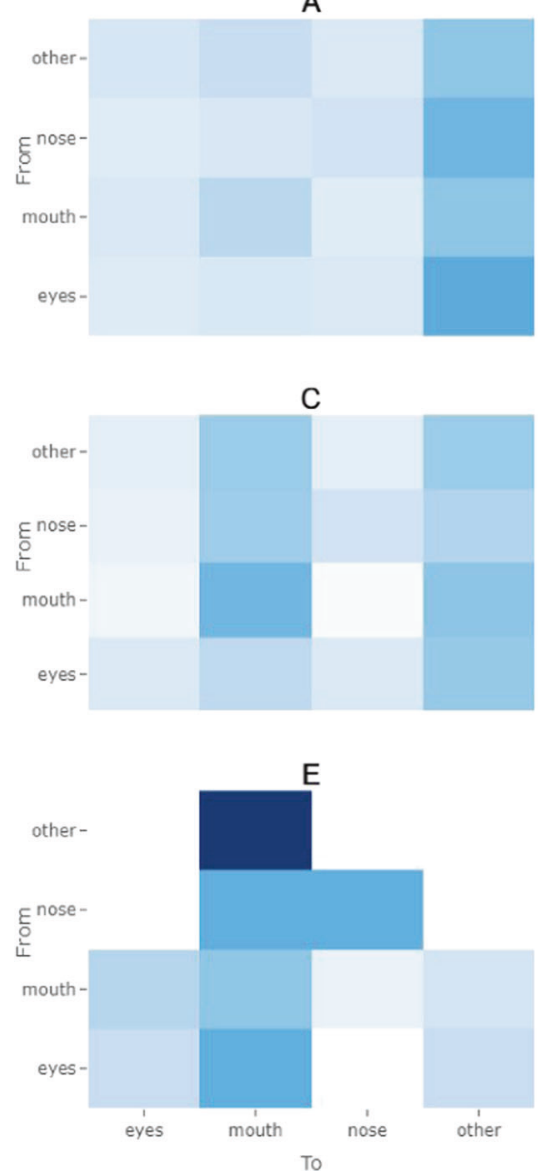

B

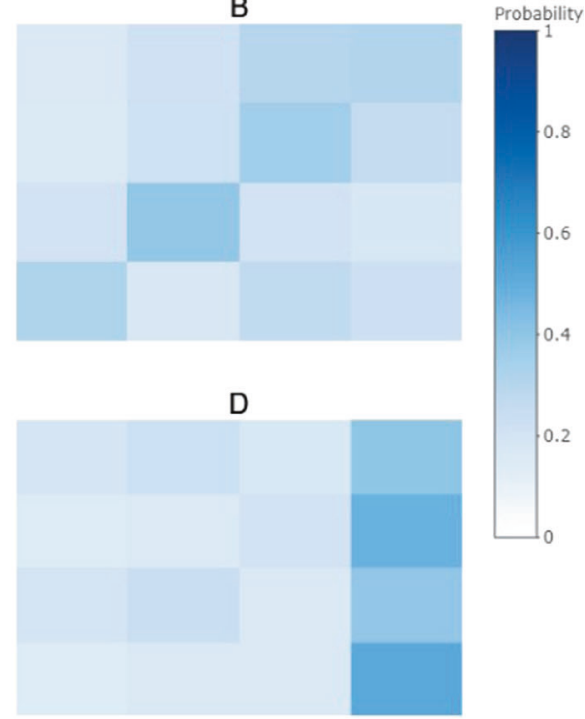

$\mathrm{F}$

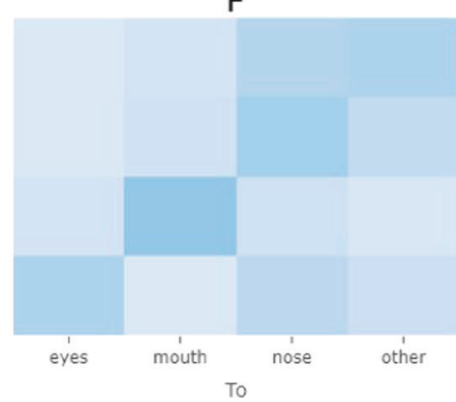

(Fig. 5a, d). In comparisons of eating vs. non-eating transitional probabilities for children, a high probability of hand-to-mouth contacts following any hand-to-head contacts, especially hand-to-other contacts, was seen (Fig. 5e). However, only three children were observed engaging in eating behaviors (Table 1). During non-eating behaviors, children appeared to make repetitive contacts with the mouth (Fig. 5f).

\section{Discussion}

\section{Key findings and generalizability}

Overall, there were no statistically significant differences between males and females other than for hand-to-nose contacts. Lack of differences between males and females is consistent with a study of 7-12 year olds where there was no statistically significant difference between male and female children in their mouthing activities [13]. However, in a study of children from 3-13 years old, females tended to touch their mouths at a greater rate than males ( $t$ test, $p=$ 0.031) [20]. In this study, children touched the mouth, eyes, and nose at a greater frequency than adults (Table 1, Fig. 2).
This is consistent with another study in which 3-4 year old children had a statistically significantly larger frequency of object-to-mouth contacts than older children of age ranges 5-6 years, 7-8 years, and 10-12 years [20].

In another study of micro-activities of adults in a graduate student office setting, Zhang et al. found that the nondominant hand did most of the facial contacts, 2.4 times more than the dominant hand. Lateralization was observed, where participants tended to use the hand on the same side of their face during contacts [21]. Differences between male and female facial contacts were not observed, potentially due to a low number of females in the study [21]. This is consistent with the findings of this study where no significant differences in contact frequency between males and females was found other than for hand-to-nose contacts (Fig. 1). Zhang et al. observed adults with a much higher hand-to-mouth contact frequency, where students touched their lips 12.8 times/h. In this study, adults during noneating behaviors touched their mouth on average 2.9 times/h (Table 1) [21]. During eating activities, adults in this study touched their mouth on average 7.7 times/h (Table 1). The largest hand-to-mouth contact frequency observed in this study for adults was 14 times/h (Table 1). In a study of ten adults in an office setting, Nicas and Best [12] found adults 
touched the lips with a mean frequency of 24 times $/ 3 \mathrm{~h}$, or 8 times/h. Zhang et al. [21] observed a larger frequency of nose touches as well, where students touched their nostrils 10.7 times $/ \mathrm{h}$, and, in this study, adults during non-eating behaviors touched their nose 2.5 times/h (Table 1) [21]. Nicas and Best [12] observed a hand-to-nose frequency of 16 times $/ 3 \mathrm{~h}$, or 5.3 times $/ \mathrm{h}$, more similar in magnitude to the hand-to-nose contact frequency observed in this study. However, Zhang et al. [21] observed a similar frequency of eye contacts, where students touched their eyes 3.9 times/h. In this study, adults during non-eating behaviors touched their eyes 2.4 times/h. Nicas and Best observed an average hand-to-eye frequency of 7.4 times $/ 3 \mathrm{~h}$, or 2.5 times $/ \mathrm{h}$. It is possible that direct observation in this study resulted in underestimates of contact frequency, while videography used by Zhang et al. [21] results in more accurate contact frequencies, as the behaviors can be reviewed multiple times and slowed down in speed, increasing the likelihood that contacts with short durations are detected [13, 22, 23]. However, the fact that participants were not informed they were being observed in the current study may mean there was less of a Hawthorne effect than in other observational methods where participants are aware of the observations. Comparisons among hand-to-face contact frequencies from this study and those from Zhang et al. [21] and Nicas and Best [12] are summarized in Table S2.

Hand-to-mouth contact frequencies are similar for the children in this study as the frequencies reported in other studies. In a meta-analysis of studies of hand-to-mouth frequency for children in indoor environments, average contact frequencies ranged from 3.9 to 20.2 contacts/h [24]. In this study, the averages of frequency of hand-to-mouth contacts for children during eating and non-eating macro-activities were 7.3 contacts/h and 4.7 contacts/h, respectively (Table 1 ). Few children were observed eating $(n=3)$, and therefore more data are needed to confirm lack of statistically significant differences in contact frequencies between adults and children during eating macro-activities (Supplementary Fig. S2).

Statistically significant differences in frequency of hand-tomouth contacts between eating and non-eating macro-activities was expected. This emphasizes the importance of hand hygiene before eating events to reduce exposures to contaminants that may be present on the hands. Statistically significant differences in frequency of hand-to-mouth, -other, and -head (all) contacts among locations were seen for observations of those not eating. Interestingly, for those eating, no statistically significant differences in contact frequency across location were seen, despite statistically significant differences in contact frequencies between eating and non-eating observations. This implies that contacts during eating behaviors are fairly consistent across location, and contacts with the mouth tended to be more frequent during eating than non-eating macro-activities for both adults and children (Table 1). For adults, frequency of contacts with the eyes and nose are similar for eating and noneating contacts. The average frequency of adults' hand-to-other contacts are slightly higher during eating macro-activities (mean $=7.6, \mathrm{SD}=4.5)$ than during non-eating macro-activities (mean $=6.2, \mathrm{SD}=3.0$ ) (Table 1). For children, hand-toeye, -nose, and -other contacts were more frequent on average during non-eating macro-activities than during eating macroactivities (Table 1). For non-eating behaviors, the significant differences in hand-to-face contact frequencies across location may be a function of changes in activities per location that then alter hand-to-face contact frequency. Future studies should evaluate connections between time activity data and hand-toface contact frequency.

\section{Applicability of micro-activities in exposure models and an illustrative example}

Incorporation of hand-to-face contact frequencies specific to adults will inform current exposure models that estimate changes in accruement of contaminants on hands based on hand-to-surface contacts and that estimate exposures via hand-to-face contacts. For example, in a model developed by Beamer et al. (2015) to estimate viral infection risks for adults based on hand-to-mouth, -eyes, and -nose contacts, the frequencies of these contact types are used to estimate total viruses transferred to facial mucosal membranes over an exposure duration $(\mathrm{T})$ (Eqs. 1, 2):

$$
\overline{C_{\text {hand }}}=\frac{\sum_{j=1}^{j=m}\left(H_{\text {surface }, j} \cdot f_{12, j}\right) \cdot C_{\text {surface }} \cdot F S A}{\alpha_{\text {die-off }}+\left(\left(\sum_{j=1}^{j=m} H_{\text {surface }}\right) \cdot f_{21} \cdot F S A\right)+\left(\frac{f_{23} \cdot \sum_{n=1}^{n=k}\left(H_{\text {orfice, }} \cdot A_{\text {orifice, },}\right)}{A_{\text {hand }}}\right)},
$$

$D=\sum_{n=1}^{k}\left(H_{\text {orifice }, n} \cdot A_{\text {orifice }, n}\right) \cdot \overline{C_{\text {hand }}} \cdot F_{23} \cdot T$.

This model has been used in other studies to estimate viral infection risks, assuming a steady state accruement of virus on the hands $[10,14]$. In this model, a steady-state concentration on hands $\left(\overline{C_{\text {hand }}}\right)$ is estimated by accounting for gains in virus to the hands due to hand-to-surface contacts and losses due to inactivation $\left(\alpha_{d i e-o f f}\right)$, transfer of virus from the hands to the surface, and transfer of the virus from the hands to the face. To estimate transfer of virus from surfaces to the hand, the frequency of hand-to-surface contacts $\left(H_{\text {surface }, j}\right)$, where $j$ represents either a porous or a nonporous surface, is multiplied by the respective surfaceto-hand transfer efficiency $\left(f_{12, j}\right)$, the concentration on the surface $\left(C_{\text {surface }}\right)$, and the fraction of total hand surface area used for the contact (FSA). To estimate transfer of virus from the hand to the surface, the frequency of hand-tosurface contacts for porous or nonporous surfaces $\left(H_{\text {surface }}\right)$ is multiplied by hand-to-surface transfer efficiency $\left(f_{21}\right)$ and 
fraction of the total hand surface area used for the contact (FSA). Due to lack of data regarding differences in hand-tosurface transfer efficiency for porous or nonporous surfaces, estimation in losses due to hand-to-surface contacts are currently not separated by surface type. However, this could easily be adjusted if the transfer efficiency data were available, similar to estimation of surface-to-hand transfer in the numerator (Eq. 1). To estimate transfer of the virus per facial orifice contact, the contact frequency $\left(H_{\text {orifice }, n}\right)$ per orifice type, $n$, is multiplied by the surface area of contact for that specific orifice type $\left(A_{\text {orific, } n}\right)$ and the hand-to-facial orifice transfer efficiency $\left(f_{23}\right)$ and then divided by the total hand surface area $\left(A_{\text {hand }}\right)$.

We can gain insights into how the frequencies presented here may inform differences in exposures for eating vs. noneating activities. In this study, adults touched their mouth an average of 7.7 times per hour while eating as opposed to 2.9 times per hour while not eating. Using Eq. (1), a difference in steady state concentration on the hands between eating and non-eating behaviors using these frequencies would depend upon other parameters affecting steady state (Eq. 1). However, an estimated dose for a fecal-oral route pathogen based on a 1-h exposure using Eq. (2) (assuming the same transfer efficiency $\left(F_{23}\right)$, steady-state concentration on hands $\left(\overline{C_{\text {hand }}}\right)$ and contact area $\left(A_{\text {orifice, }}\right)$ ) would mean a dose while eating would be approximately 2.7 times larger during eating than non-eating. If we consider a virus that can be transmitted via hand-to-mouth, -eyes, or -nose contacts, such as SARS-CoV-2, and use average adult hand-toface contact frequencies from this study, an hour of eating behaviors would result in 12.2 contacts that could result in a dose (mouth: 7.7, eyes: 2.1, nose: 2.4) and 7.8 contacts (mouth: 2.9, eyes: 2.4, nose: 2.5 ) for non-eating behaviors. This would result in approximately a 1.6 times greater dose for eating behaviors than non-eating behaviors. Because dose-response curves relating doses to infection risks are typically non-linear, small changes in dose could have large implications for infection risks, especially in the case of highly infectious viruses with low infectious doses.

While the frequency of hand-to-face and other types of hand-to-object contacts are often included in exposure models, the order in which surfaces are contacted is not always incorporated. However, sequences of behaviors may vary from one type of activity to another, informing how exposures may vary from one activity to another, especially when handwashing or other hand hygiene events are taken into account. In healthcare, discrete Markov chains have been used to simulate sequences of hand-to-surface contacts for healthcare professionals and subsequent spread of pathogens around patient rooms [25]. In chemical contexts, Markov chains were also used to simulate "contact events" [26]. To our knowledge, sequences of hand-to-face contacts, specifically, have not been incorporated in current microbial exposure models. It is unknown what patterns of hand-to-face contacts may mean for infection risk. Future studies should evaluate methods for incorporating discrete Markov chains describing sequences of hand-to-face contacts and other behaviors, such as contacts with surfaces and handwashing events, into current exposure models. It should then be evaluated how these patterns influence estimated exposures or health outcomes.

Duration is also rarely incorporated in microbial exposure models, despite availability of duration data for handto-surface and -mouth contacts [13]. It has been addressed in chemical contacts, especially in estimating dermal exposures and considering the absorption of chemicals on the skin over the duration of different contact events [27]. In microbial contexts, this may be due to the fact that models currently do not calculate changes in microbial concentration differently for a one second contact vs. a contact of longer or shorter duration. Mechanistic models describing the effect of contact time on transfer efficiency are needed to more accurately capture fomite-mediated exposures and microbial accruement on hands. Very few data are available describing microbial transfer for hand-to-mouth contacts [28], and, to our knowledge, no data are yet available for hand-to-eye or -nose contacts. These data would be needed to evaluate the relative importance of hand-to-mouth vs. -eye or -nose contacts to exposure and subsequent infection risk, along with an understanding of how durations of these contacts increases transfer and therefore chemical risks and infection risks from pathogens.

\section{Limitations}

There was one observer in this study. While this may mean there was consistency from one observation to the next, this also means that only one person's perception of the behaviors is represented by these data, potentially introducing an observer bias. This has occurred in other micro-activity studies, even in which videography data was collected where there is sometimes one translator [29]. In the case of this study, because the observer has since passed away, their specific insights on observations are unfortunately unavailable. While translation of observational form data to a digital spreadsheet was spot checked by multiple researchers, double coding and comparison of the coding of data from observational forms would have been more robust. Another limitation of this study was that the observations were conducted in 2001. With notable changes in the types of activities conducted in a variety of locations, such as contacts with cell phones and other technological devices, it is possible that contact behaviors with the face may have changed. For example, when one or both hands are occupied with a cellular device, they are not as readily available for a hand-to-face contact than if the hands were empty or 
holding something that one was not actively using. Whether time on cell phones has decreased or altered the frequency of hand-to-face contacts is unknown. This study may serve as a baseline of hand-to-face contact frequency before the wider implementation of mobile and touch screen devices, and future research is needed to evaluate the effects of technological surfaces on hand-to-face and hand-to-surface contact frequencies.

Participants in this study were observed in public spaces. To avoid the Hawthorne effect [30], participants were not informed that they were being observed. This resulted in some data being unconfirmed, such as age or culture. It is possible that differences in contact frequency among different age groups or cultures are present that are not currently being accounted for. For example, in other studies, the effect of age on contact frequency has been observed, especially for children where age has been shown to be negatively correlated with some hand-to-surface contacts [13]. While differences in object-to-mouth and hand-to-mouth contact frequency for children living in Taiwan versus the United states have been evaluated [31], the effects of age and/or culture on contact frequency for adults, specifically, are unknown.

In addition, it has been demonstrated that mouthing behaviors may be significantly different between indoor and outdoor environments, with greater frequency indoors than outdoors [24]. In this study, it was unknown whether some of the recorded locations were indoors, outdoors, or a hybrid, such as the food court, zoo, or museum. We were therefore unable to confidently compare behavioral differences between indoor and outdoor locations. This further reiterates the limitation of direct observation, where videography data would allow future researchers to revisit observations and confirm specific data [13, 22]. This limitation aside, our comparisons among all locations indicate that contact frequencies do vary from one location to another (Fig. 4 and Supplementary Fig. S3). However, observations in each location did not span multiple seasons. It is possible there are seasonal effects of frequency of handto-face contacts that are not accounted for in this study. For example, colder seasons could result in a runny nose, and, therefore, a greater frequency of hand-to-nose contacts. The effect of season could also depend upon the geographical location. Future data are needed, ideally collected with videography methods, to determine whether indoor vs. outdoor characteristics explain differences in contact frequencies between locations, after adjusting for effects of geographical location and season.

\section{Conclusions}

To our knowledge, this is the first study to report hand-toface, -eyes, and -nose contacts for adults in multiple locations and hand-to-eye and -nose frequencies for children. These data can inform assumed hand-to-face frequencies and even contact sequences in future risk assessments, reducing uncertainty in one of the most influential categories of inputs in the case of several chemical and microbial risk assessments. More data are needed to investigate differences in contact frequency among age groups and different cultures for adults and to further investigate the modification of hand-to-face contact frequencies during eating vs. non-eating macroactivities.

\section{Data availability}

Data are available at https://doi.org/10.5518/798.

\section{Code availability}

Codes are available at https://doi.org/10.5518/798.

Acknowledgements The authors would like to acknowledge Patricia A. Rusin, $\mathrm{PhD}$, the researcher who collected the data, and the people whom she observed. AMW was supported by the University of Arizona Foundation and the Hispanic Women's Corporation/Zuckerman Family Foundation Student Scholarship Award through the Mel and Enid Zuckerman College of Public Health, University of Arizona. PIB was supported by NIEHS P30 ES006694. M-FK was supported by the Engineering and Physical Sciences Research Council, UK: Healthcare Environment Control, Optimization and Infection Risk Assessment (HECOIRA.leeds.ac.uk) (EP/PO/23312/1) and the Leeds Institute for Fluid Dynamics International Mobility Grant.

\section{Compliance with ethical standards}

Conflict of interest The authors declare that they have no conflict of interest.

Publisher's note Springer Nature remains neutral with regard to jurisdictional claims in published maps and institutional affiliations.

Open Access This article is licensed under a Creative Commons Attribution 4.0 International License, which permits use, sharing, adaptation, distribution and reproduction in any medium or format, as long as you give appropriate credit to the original author(s) and the source, provide a link to the Creative Commons license, and indicate if changes were made. The images or other third party material in this article are included in the article's Creative Commons license, unless indicated otherwise in a credit line to the material. If material is not included in the article's Creative Commons license and your intended use is not permitted by statutory regulation or exceeds the permitted use, you will need to obtain permission directly from the copyright holder. To view a copy of this license, visit http://creativecommons. org/licenses/by/4.0/.

\section{References}

1. Sahmel J, Hsu EI, Avens HJ, Beckett EM, Devlin KD. Estimation of hand-to-mouth transfer efficiency of lead. Ann Work Expo Heal. 2015;59(2):210-20. 
2. Beamer PI, Canales RA, Ferguson AC, Leckie JO, Bradman ASA. Relative pesticide and exposure route contribution to aggregate and cumulative dose in young farmworker children. Int J Environ Res Public Health. 2012;9:73-96.

3. Hendley JO, Wenzel RP, Gwaltney JM. Transmission of rhinovirus colds by self-inoculation. N Engl J Med. 1973;288(26):1361-4.

4. Belser JA, Gustin KM, Maines TR, Pantin-Jackwood MJ, Katz JM, Tumpey TM. Influenza virus respiratory infection and transmission following ocular inoculation in ferrets. PLoS Pathog. 2012;8(3):e1002569.

5. Killingley B, Nguyen-Van-Tam J. Routes of influenza transmission. Influenza Other Respir Viruses. 2013;7(Suppl. 2):42-51.

6. Kampf G, Todt D, Pfaender S, Steinmann E. Persistence of coronaviruses on inanimate surfaces and its inactivation with biocidal agents. J Hosp Infect. 2020;104(3):246-51.

7. Beamer PI, Plotkin KR, Gerba CP, Sifuentes LY, Koenig DW, Reynolds KA. Modeling of human viruses on hands and risk of infection in an office workplace using micro-activity data. J Occup Environ Hyg. 2015;12(4):266-75.

8. Canales RA, Reynolds KA, Wilson AM, Fankem SLM, Weir MH, Rose JB, et al. Modeling the role of fomites in a norovirus outbreak. J Occup Environ Hyg. 2019;16(1):16-26. https://doi.org/ 10.1080/15459624.2018.1531131

9. Canales RA, Wilson AM, Sinclair RG, Soto-Beltran M, PearceWalker J, Molina M, et al. Microbial study of household hygiene conditions and associated Listeria monocytogenes infection risks for Peruvian women. Trop Med Int Health. 2019;24(7):899-921. https://onlinelibrary.wiley.com/doi/abs/10.1111/tmi.13246

10. Wilson AM, Reynolds KA, Sexton JD, Canales RA. Modeling surface disinfection needs to meet microbial risk reduction targets. Appl Environ Microbiol. 2018;84(18):e00709-18.

11. Julian TR, Canales RA, Leckie JO, Boehm AB. A model of exposure to rotavirus from nondietary ingestion iterated by simulated intermittent contacts. Risk Anal. 2009;29(5):617-32.

12. Nicas M, Best D. A study quantifying the hand-to-face contact rate and its potential application to predicting respiratory tract infection. J Occup Environ Hyg. 2008;5(6):347-52.

13. Beamer PI, Luik CE, Canales RA, Leckie JO. Quantified outdoor micro-activity data for children aged 7-12-years old. J Expo Sci Environ Epidemiol. 2012;22(1):82-92. https://doi.org/10.1038/ jes.2011.34

14. Contreras RD, Wilson AM, Garavito F, Sexton JD, Reynolds KA, Canales RA. Assessing virus infection probability in an office setting using stochastic simulation. J Occup Environ Hyg. 2020;17(1):30-7. https://www.tandfonline.com/doi/full/10.1080/ 15459624.2019.1691219

15. Wilson R, Jones-Otazo H, Petrovic S, Mitchell I, Bonvalot Y, Williams $\mathrm{D}$, et al. Revisiting dust and soil ingestion rates based on hand-to-mouth transfer. Hum Ecol Risk Assess. 2013;19(1):158-88.

16. U.S. Environmental Protection Agency. Exposure factors handbook 2011 Edition (EPA/600/R-09/052F) [Internet]. Washington, DC; 2011. https://cfpub.epa.gov/ncea/risk/recordisplay.cfm?deid $=236252$
17. Kwok YL, Gralton J, McLaws ML. Face touching: a frequent habit that has implications for hand hygiene. Am J Infect Control. 2015;43(2):112-4. https://doi.org/10.1016/j.ajic.2014.10.015

18. Landrigan PJ, Kimmel CA, Correa A, Eskenazi B. Children's health and the environment: public health issues and challenges for risk assessment. Environ Health Perspect. 2004;112(2):257-65.

19. Spedicato G. Discrete Time Markov Chains with R. R J. 2017.

20. Freeman NC, Jimenez M, Reed KJ, Gurunathan S, Edwards RD, Roy A, et al. Quantitative analysis of children's microactivity patterns: the Minnesota Children's Pesticide Exposure Study. J Expo Anal Environ Epidemiol. 2001;11(6):501-9.

21. Zhang N, Wang P, King M-F, Chan P-T, Li Y. Most self-touches are with the nondominant hand. Sci Rep. 2020;10(1):10457.

22. Cohen Hubal EA, Sheldon LS, Burke JM, McCurdy TR, Berry MR, Rigas ML, et al. Children's exposure assessment: a review of factors influencing children's exposure, and the data available to characterize and assess that exposure. Environ Health Perspect. 2000;108(6):475-86.

23. Ferguson AC, Canales RA, Beamer P, Auyeung W, Key M, Munninghoff A, et al. Video methods in the quantification of children's exposures. J Expo Sci Environ Epidemiol. 2006;16 (3):287-98. http://www.nature.com/doifinder/10.1038/sj.jea.7500459

24. Xue J, Zartarian V, Moya J, Freeman N, Beamer P, Black K, et al. A meta-analysis of children's hand-to-mouth frequency data for estimating nondietary ingestion exposure. Risk Anal. 2007;27 (2):411-20.

25. King MF, Noakes CJ, Sleigh PA. Modeling environmental contamination in hospital single- and four-bed rooms. Indoor Air. 2015;25(6):694-707.

26. Canales RA. The cumulative and aggregate simulation of exposure framework. Stanford, CA: Stanford University; 2004. Ph.D. Dissertation.

27. Zartarian VG, Ozkaynak H, Burke JM, Zufall MJ, Rigas ML, Furtaw EJ Jr. A modeling framework for estimating children's residential exposure and dose to chlorpyrifos via dermal residue contact and nondietary ingestion. Environ Health Perspect. 2000; 108(6):505-14.

28. Rusin P, Maxwell S, Gerba C. Comparative surface-to-hand and fingertip-to-mouth transfer efficiency of gram-positive bacteria, gram-negative bacteria, and phage. J Appl Microbiol. 2002;93 (4):585-92.

29. Tsou MC, Özkaynak H, Beamer P, Dang W, Hsi HC, Bin JiangC, et al. Mouthing activity data for children age 3 to $<6$ years old and fraction of hand area mouthed for children age $<6$ years old in Taiwan. J Expo Sci Environ Epidemiol. 2018;28(2):182-92.

30. Goodwin MA, Stange KC, Zyzanski SJ, Crabtree BF, Borawski EA, Flocke SA. The Hawthorne effect in direct observation research with physicians and patients. J Eval Clin Pract. 2017;23 (6):1322-8.

31. Tsou MC, Özkaynak H, Beamer P, Dang W, Hsi HC, Bin JiangC, et al. Mouthing activity data for children aged 7 to 35 months in Taiwan. J Expo Sci Environ Epidemiol. 2015;25(4):388-98. 